\title{
NONLOCAL AND MULTIVARIATE MATHEMATICAL MORPHOLOGY
}

\author{
O. Lézoray, A. Elmoataz \\ Université de Caen Basse-Normandie, GREYC - UMR CNRS 6072, Caen, FRANCE
}

\begin{abstract}
The generalization of mathematical morphology to multivariate images is addressed in this paper. The proposed approach is fully unsupervised and consists in constructing a complete lattice from an image as a rank transformation together with a learned ordering of vectors. This unsupervised ordering of vectors relies on three steps: dictionary learning, manifold learning and out of sample extension. In addition to providing an efficient way to construct a vectorial ordering, nonlocal configurations based on color patches can be easily handled and provide much better results than with classical local morphological approaches.
\end{abstract}

Index Terms - Mathematical morphology, nonlocal, multivariate, manifold learning.

\section{INTRODUCTION}

Mathematical Morphology (MM) is a nonlinear approach to image processing that relies on a fundamental structure, the complete lattice $(\mathcal{L}, \leq)$ [1]. The construction of morphological operators therefore needs an ordering between the elements to be processed. Theoretically, a partial ordering is sufficient to construct complete lattices. However, total orderings are preferred since they are vector preserving [2]. With the acceptance of complete lattice theory, it is possible to define morphological operators for any type of multivariate image data once a proper ordering is established [2]. However, if MM is well defined for binary and gray scale images, there exist no general admitted extension that permits to perform morphological operations on multivariate data since there is no natural ordering on vectors. Therefore, the extension of Mathematical Morphology to multivariate images is a very active field. Many different approaches have been proposed in literature for color and hyperspectral images [2, 3, 4]. This paper introduces a systematic approach towards the construction of complete lattices for any kind of multivariate data. Following recent approaches $[3,4]$, we propose to use unsupervised manifold learning to construct complete lattices on multivariate data by considering the theoretical framework of $h$-orderings [5]. We also consider the case of associating patches vectors to pixels and show how our proposal can be naturally used to obtain a innovative nonlocal formulation of morphological operators.

\section{COMPLETE LATTICES IN $\mathbb{R}^{P}$}

A complete lattice $\mathcal{L}$ is a non-empty set equipped with a (partial or total) ordering relation, such that every non-empty subset $\mathcal{P}$ of $\mathcal{L}$ has a lower bound $\wedge \mathcal{P}$ and an upper bound $\vee \mathcal{P}$. In this context, images are modeled by functions mapping their domain space $\Omega$, into a complete lattice $\mathcal{L}$. Within this model, morphological operators are represented as mappings between complete lattices in combination with matching patterns called structuring elements that are subsets of $\Omega$.

In the sequel, we consider the general case of multivariate images. A multivariate image can be represented by the mapping $f: \Omega \subset \mathbb{Z}^{l} \rightarrow \mathcal{T} \subset \mathbb{R}^{p}$ where $l$ is the image dimension, $p$ the number of channels, and $\mathcal{T}$ is a non-empty set. One way to define an ordering relation between vectors of $\mathcal{T}$ is to use the framework of $h$-orderings [5]. This corresponds to defining a surjective transform $h$ from $\mathcal{T}$ to $\mathcal{L}$ where $\mathcal{L}$ is a complete lattice equipped with the conditional total ordering [5]. With $h: \mathcal{T} \rightarrow \mathcal{L}$ and $\mathbf{x} \rightarrow h(\mathbf{x}), \forall\left(\mathbf{x}_{i}, \mathbf{x}_{j}\right) \in \mathcal{T} \times \mathcal{T}$, $\mathbf{x}_{i} \leq_{h} \mathbf{x}_{j} \Leftrightarrow h\left(\mathbf{x}_{i}\right) \leq h\left(\mathbf{x}_{j}\right)$. Then, $\mathcal{T}$ is no longer required to be a complete lattice, since the ordering of $\mathcal{L}$ can be induced upon $\mathcal{T}$ by means of $h$ [2]. When $h$ is bijective, this corresponds to define a space filling curve that goes through each point of the set $\mathcal{T}$ just once and thus induces a total ordering. Therefore, there is an equivalence: (total ordering on $\mathcal{T}) \Leftrightarrow$ (bijective application $h: \mathcal{T} \rightarrow \mathcal{L}$ ) $\Leftrightarrow$ (space filling curve in $\mathcal{T}$ ) [2]. Moreover, we previously shown that another equivalence can be considered [4]: (total ordering on $\mathcal{T}) \Leftrightarrow($ rank transformation on $\mathcal{T}$ ). Indeed, since a total $h$-ordering $\leq_{h}$ orders all the vectors of the set $\mathcal{T}$, it is possible to sort all these vectors and obtain their rank in the ordering, creating explicitly the complete lattice $\left(\mathcal{T}, \leq_{h}\right)$ [4]. Once the complete lattice is created, each element of the multivariate image can be replaced by its rank, creating a rank image. This (scalar) rank image is the lattice representation of the multivalued image according to the ordering strategy $\leq_{h}$.

\section{COMPLETE LATTICE LEARNING}

Usual approaches to mathematical morphology do not explicitly construct the complete lattice: they first define a total ordering relation (usually a specific lexicographic ordering) that induces a complete lattice. In this paper, we take an opposite approach and we explicitly learn the complete lattice 
from a multivariate image $f: \Omega \rightarrow \mathbb{R}^{p}$ using $h$-ordering $h: \mathbb{R}^{p} \rightarrow \mathcal{L}$. It is obvious that $h$ cannot be linear since a distortion of the space topology is inevitable. As a consequence, we choose to focus our developments on manifold learning to construct $h$. Obviously, constructing the complete lattice of an image with manifold learning directly from all the pixels is computationally unfeasible. Therefore, we propose a three-step strategy towards construct the $h$-ordering.

\subsection{Data Quantization}

Since the complexity of manifold learning is highly dependent on the number of input data, we first reduce the amount of data of a multivariate image by Vector Quantization (VQ). VQ maps a vector $\mathbf{x}$ to another vector $\mathbf{x}^{\prime}$ that belongs to $n$ prototype vectors the set of which is named a dictionary. A dictionary $\mathcal{D}$ is built from a training set $I$ of size $m(m \gg n)$. A VQ algorithm has to produce a set $\mathcal{D}$ of prototypes $\mathbf{x}^{\prime}$ that minimizes the distortion defined by $\frac{1}{m} \sum_{i=1}^{m} \min _{1 \leq j \leq n}\left\|\mathbf{x}_{i}-\mathbf{x}_{j}^{\prime}\right\|_{2}$. LBG [6] is one algorithm that can build such a dictionary. It is an iterative algorithm that produces $n=2^{k}$ prototypes after $k$ iterates. Given a multivariate image of $m$ pixels, VQ is applied to construct a dictionary $\mathcal{D}=\left\{\mathbf{x}_{1}^{\prime}, \ldots, \mathbf{x}_{n}^{\prime}\right\}$ where $\mathbf{x}_{i}^{\prime} \in \mathbb{R}^{p}$.

\subsection{Manifold learning}

Once the dictionary $\mathcal{D}$ is obtained, we construct the transformation $h$ on $\mathcal{D}$ with manifold learning. Manifold learning is the counterpart to Principal Component Analysis which aims at finding a low dimensional parametrization for data sets that lie on nonlinear manifolds in a high-dimensional space. In the last few years, many manifold learning algorithms have been proposed that share the use of an eigen-decomposition for obtaining a lower-dimensional embedding of the data. In this paper, we choose to use Laplacian Eigenmaps [7]. Let $\left\{\mathbf{x}_{1}^{\prime}, \cdots, \mathbf{x}_{n}^{\prime}\right\}$ with $\mathbf{x}_{i}^{\prime} \in \mathbb{R}^{p}$ be the $n$ vectors of the dictionary $\mathcal{D}$. Manifold learning consists in searching for a new representation $\left\{\mathbf{y}_{1}, \cdots, \mathbf{y}_{n}\right\}$ with $\mathbf{y}_{i} \in \mathbb{R}^{n}$. One starts by computing a similarity matrix $W$ that contains the pairwise similarities between all the input vectors $\mathbf{x}_{i}^{\prime}: W_{i j}=k\left(\mathbf{x}_{i}^{\prime}, \mathbf{x}_{j}^{\prime}\right)=$ $e\left(-\frac{\left\|\mathbf{x}_{i}^{\prime}-\mathbf{x}_{j}^{\prime}\right\|^{2}}{\sigma^{2}}\right)$. To have a parameter free algorithm, $\sigma$ is set to the maximum distance between the vectors of the dictionary. The degree diagonal matrix is denoted by $D$ with $D_{i i}=\sum_{j} W_{i j}, L=D-W$ is the Laplacian matrix and $\tilde{L}$ is the normalized Laplacian defined by $\tilde{L}=D^{-\frac{1}{2}} L D^{-\frac{1}{2}}=$ $I-D^{-\frac{1}{2}} W D^{-\frac{1}{2}}$. Laplacian Eigenmaps manifold learning consists in searching for a new representation obtained by minimizing $\frac{1}{2} \sum_{i j}\left\|\mathbf{y}_{i}-\mathbf{y}_{j}\right\|_{2} W_{i j}=\operatorname{Tr}\left(\mathbf{Y}^{T} \tilde{L} \mathbf{Y}\right)$ with $\mathbf{Y}=$ $\left[\mathbf{y}_{1}, \cdots, \mathbf{y}_{n}\right]$. This cost function encourages nearby sample vectors to be mapped to nearby outputs. This is achieved by finding the eigenvectors $\mathbf{y}_{1}=\phi_{1}, \cdots, \mathbf{y}_{n}=\phi_{n}$ of matrix
$\tilde{L}$. A low-dimensional representation is obtained by considering the $q$ lowest eigenvectors (the first eigenvector being discarded) with $q \ll p$ and is defined by the following operator $h_{\mathcal{D}}: \mathbf{x}_{i}^{\prime} \rightarrow\left(\phi_{2}\left(\mathbf{x}_{i}^{\prime}\right), \cdots, \phi_{q}\left(\mathbf{x}_{i}^{\prime}\right)\right)^{T}$ where $\phi_{k}\left(\mathbf{x}_{i}^{\prime}\right)$ is the $i^{\text {th }}$ coordinate of eigenvector $\phi_{k}$. This obtained projection operator corresponds to constructing a $h$-ordering.

\subsection{Out of sample extension}

To dispose of a complete lattice, we have to define the projection $h$ on all the vectors of the image and not only its dictionary with $h_{\mathcal{D}}$. The dictionary $\mathcal{D}$ being a sub-manifold of the complete lattice, we need to extend eigenfunctions computed on the dictionary to new unexplored vectors from the original image. This can be achieved by the Nyström method that interpolates the value of eigenvectors computed on $n$ sample vectors $\mathbf{x}_{i}^{\prime}$ to $m$ novel vectors $\mathbf{x}_{i}$. To extrapolate a new vector $\mathbf{x}$, the Nyström estimator with $n$ samples for the $k$-th eigenvector is

$$
\phi_{k}\left(\mathbf{x}_{j}\right)=\frac{1}{\lambda_{k}} \sum_{i=1}^{n} \phi_{k}\left(\mathbf{x}_{i}^{\prime}\right) k\left(\mathbf{x}_{j}, \mathbf{x}_{i}^{\prime}\right)
$$

where $\lambda_{k}$ is the $k$-th eigenvalue of the normalized Laplacian $\tilde{L}$ and $\phi_{k}\left(\mathbf{x}_{i}^{\prime}\right)$ is the $i$-th element of the $k$-th eigenvector of $\tilde{L}$. Let us instantiate Equation (1) in the context of the normalized Laplacian. First, note that if $\lambda_{k}$ is an eigenvalue of of $L$, then $1-\lambda_{k}$ is an eigenvalue of $D^{-\frac{1}{2}} W D^{-\frac{1}{2}}$. Applying the Nyström extension to compute the eigenvectors of the normalized Laplacian $\tilde{L} \phi_{k}=\lambda_{k} \phi_{k}$, we get

$$
\phi_{k}\left(\mathbf{x}_{j}\right)=\frac{1}{1-\lambda_{k}} \sum_{i=1}^{n} \frac{k\left(\mathbf{x}_{j}, \mathbf{x}_{i}^{\prime}\right)}{\sqrt{d\left(\mathbf{x}_{j}\right) d\left(\mathbf{x}_{i}^{\prime}\right)}}
$$

where $d(\mathbf{x})=\sum_{i=1}^{n} k\left(\mathbf{x}, \mathbf{x}_{i}^{\prime}\right)$. With this formulation, we are now in position to compute the projection $h$ for any pixel of the image.

\subsection{Complete lattice construction}

With these three sequential ingredients, we can now construct a rank transformation that expresses explicitly the complete lattice of the vectors of a multivariate image. Given a multivariate image $f: \Omega \rightarrow \mathbb{R}^{p}$ that provides a set $I=\left\{\mathbf{x}_{1}, \cdots, \mathbf{x}_{m}\right\}$ of $m$ vectors in $\mathbb{R}^{p}$, a dictionary $\mathcal{D}=\left\{\mathbf{x}_{1}^{\prime}, \cdots, \mathbf{x}_{n}^{\prime}\right\}$ of $n$ vectors in $\mathbb{R}^{p}$ is computed. Manifold learning is performed on the dictionary and a new representation $h_{\mathcal{D}}$ is obtained for each element of the dictionary. This new representation is interpolated to all the pixels of the image with the Nyström out of sample extension, defining $h: \mathbb{R}^{p} \rightarrow \mathbb{R}^{q}$ as $h(\mathbf{x})=\left(\phi_{2}(\mathbf{x}), \cdots, \phi_{q}(\mathbf{x})\right)^{T}$. Once this representation is obtained, the complete lattice $\left(\mathbb{R}^{p}, \leq_{h}\right)$ can be explicitly constructed as well as the rank transformation. First, we sort all vectors of $f$ according to $\leq_{h}$ (the conditional total ordering on $h(\mathbf{x})$ ) and obtain a sorted 
image $f_{s}$. This sorted image $f_{s}:[0, m] \rightarrow \mathbb{R}^{p}$ defines the ordering of the vectors of $f$. From this ordering, we can deduce the rank of a vector on the complete lattice $\mathcal{L}$ defined as $r: \mathbb{R}^{q} \rightarrow[0, m]$, and construct a rank image as $f_{r}: \Omega \rightarrow[0, m]$ with $f_{r}\left(p_{i}\right)=(r \circ h \circ f)\left(p_{i}\right)$ and $p_{i} \in \Omega$. With these elements, the original image is now represented by the rank image $f_{r}$ and the ordering of the pixels' vectors $f_{s}$. The original image is recovered exactly since $f\left(p_{i}\right)=\left(f_{s} \circ f_{r}\right)\left(p_{i}\right)$. Given a specific morphological processing $g$, the corresponding processed multivariate image is obtained by $g\left(f\left(p_{i}\right)\right)=\left(f_{s} \circ g \circ f_{r}\right)\left(p_{i}\right)$.

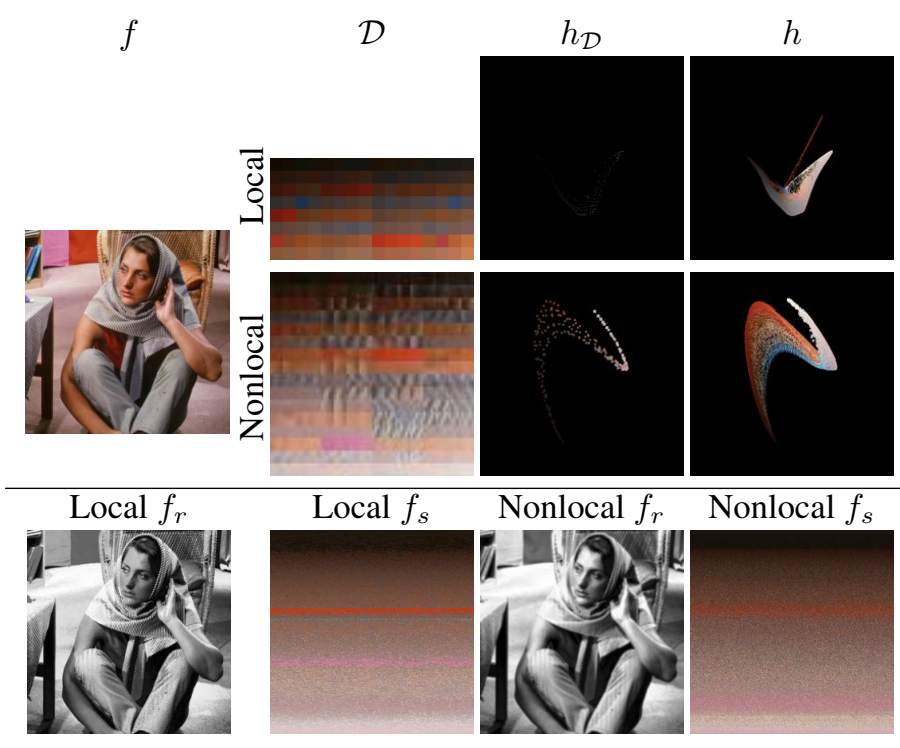

Fig. 1. Illustration of the proposed approach. First part, from left to right: the standard Barbara image $f$, the dictionary $\mathcal{D}(n=256)$, the manifold $h_{\mathcal{D}}$ learned from the dictionary, the manifold $h$ interpolated to all the image vectors. Second part: the rank image $f_{r}$ and the sorted vectors $f_{s}$ in local and nonlocal configurations

\section{APPLICATION TO LOCAL AND NONLOCAL MORPHOLOGICAL PROCESSING}

The approach we propose is easily applicable to any kind of multivariate images even if we only provide illustrations with colors images. Figure 1 illustrates the proposed approach. The image is first quantized into $n=256$ colors and manifold learning is performed on this dictionary $\mathcal{D}$ to obtain a new representation $h_{\mathcal{D}}$. The latter is extended to all the original colors of $f$ to construct the global manifold learning representation $h$. From this, the rank image $f_{r}$ and the sorted set of vectors $f_{s}$ are obtained. The rank image is a grayscale image of $m$ levels that can be directly used for any classical morphological processing.

Recently, nonlocal schemes for image processing have received a lot of attention [8]. Rather than considering only the vector associated to one pixel to compute pixel similarities, patches around these pixels are considered. These patches capture the dependencies of neighboring pixels and thus can distinguish textural patterns. In previous works [9], we extended PDEs-based morphology to nonlocal processing on images represented by proximity graphs. On the roots of our works, [10] proposed some nonlocal pseudo-morphological operators that make use of a nearest neighbors graph. If innovative, none of these works enables to extend the classical flat algebraic morphological operators to nonlocal patch configurations. On the opposite, our approach directly enables it.

Given a color image $f: \Omega \rightarrow \mathbb{R}^{3}$, a patch of size $w^{2}$ represented as a vector of size $3 w^{2}$ is associated to each pixel giving the image $f^{\prime}: \Omega \rightarrow \mathbb{R}^{3 w^{2}}$. On this image, the complete lattice creation is performed. This enables to construct the rank image according to the Manifold where patche lives which is highly nonlinear. Moreover, since the complete lattice is constructed according to patch similarities and not single pixel colors, the textured parts of the image are better captured and the complete lattice has a much better $h$-ordering. Once the rank image $f_{r}$ is obtained, this gives a nonlocal (but scalar) representation of the image that can be used for morphological processing. Finally, to be able to exactly reconstruct the original image $f$, the image of sorted vectors $f_{s}$ stores the color vectors and not the pixel patches.

Figure 1 also illustrates the nonlocal proposed approach. The image $f^{\prime}$ is quantized into $n=256$ patches and a new representation $h_{\mathcal{D}}$ is obtained by manifold learning. The latter is then extended to the whole set of pixel patches. As it can be seen, the ordering of the colors vectors $f_{s}$ is very different between local and nonlocal configurations. The rank image is smoother and much more homogeneous in regions of similar texture assessing the interest in the use of color patches information instead of color.

Figure 2 illustrates the interest of the proposed approach on two textured images. First part of figure 2 presents the obtained rank images $f_{r}$ in local (color vectors) or nonlocal (color patches) configurations. Pixels described by similar (color or patch) vectors have close ranks. However, the nonlocal rank image better exhibit similar textures and is much less sensitive to small variations. Second and third parts of figure 2 present morphological operator results. As it can be seen on figure 2, the benefit of nonlocal configurations is evident. For basic morphological operations such as erosion, dilation and closing, nonlocal configurations enable to better preserve textured parts of the image while simplifying it. With morphological gradient and white top hat, the advantage is really put forward and the nonlocal approach enables to better exhibit salient edges in the original image. Asa consequence, this permits to obtain better watershed segmentation results. 


\section{CONCLUSION}

This paper introduces an unsupervised approach towards the construction of complete lattices for multivariate images and consequently a framework for unsupervised multivariate mathematical morphology. In contrast to usual approaches, no prior information (e.g., component prioritization) is required. The approach relies on dictionary learning, manifold learning and out of sample extension. In addition to providing a general approach to multivariate mathematical morphology, we also introduce novel nonlocal flat algebraic mathematical morphology operators that have never been investigated before.

\section{REFERENCES}

[1] C. Ronse, "Why mathematical morphology needs complete lattices," Signal Process., vol. 21, no. 2, pp. 129154, 1990.

[2] E. Aptoula and S. Lefèvre, "Multivariate mathematical morphology applied to colour image analysis," in Multivariate image processing: methods and applications, C. Collet, J. Chanussot, and K. Chehdi, Eds., pp. 303337. ISTE - John Wiley, 2009.

[3] S. Velasco-Forero and J. Angulo, "Supervised ordering in $\mathbb{R}^{p}$ : Application to morphological processing of hyperspectral images," IEEE Trans. Image Process., vol. 20, no. 11, pp. 3301-3308, 2011.

[4] O. Lezoray, C. Charrier, and A. Elmoataz, "Rank transformation and manifold learning for multivariate mathematical morphology," in EUSIPCO, 2009.

[5] J. Goutsias, H.J.A.M. Heijmans, and K. Sivakumar, "Morphological operators for image sequences," Comput. Vision Image Understanding, vol. 62, no. 3, pp. 326-346, 1995.

[6] A. Gersho and R.M. Gray, Vector Quantization and Signal Compression, Kluwer Academic, 1991.

[7] M. Belkin and P. Niyogi, "Laplacian eigenmaps for dimensionality reduction and data representation," Neural Comput., vol. 15, no. 6, pp. 1373-1396, 2003.

[8] A. Buades, B. Coll, and J.-M. Morel, "Image denoising methods. a new nonlocal principle," SIAM Review, vol. 52, no. 1, pp. 113-147, 2010.

[9] V.T. Ta, A. Elmoataz, and O. Lézoray, "Nonlocal pdesbased morphology on weighted graphs for image and data processing," IEEE Trans. Image Process., vol. 20, no. 6, pp. 1504-1516, June 2011.

[10] P. Salembier, "Study on nonlocal morphological operators," in ICIP, 2009, pp. 2269-2272.
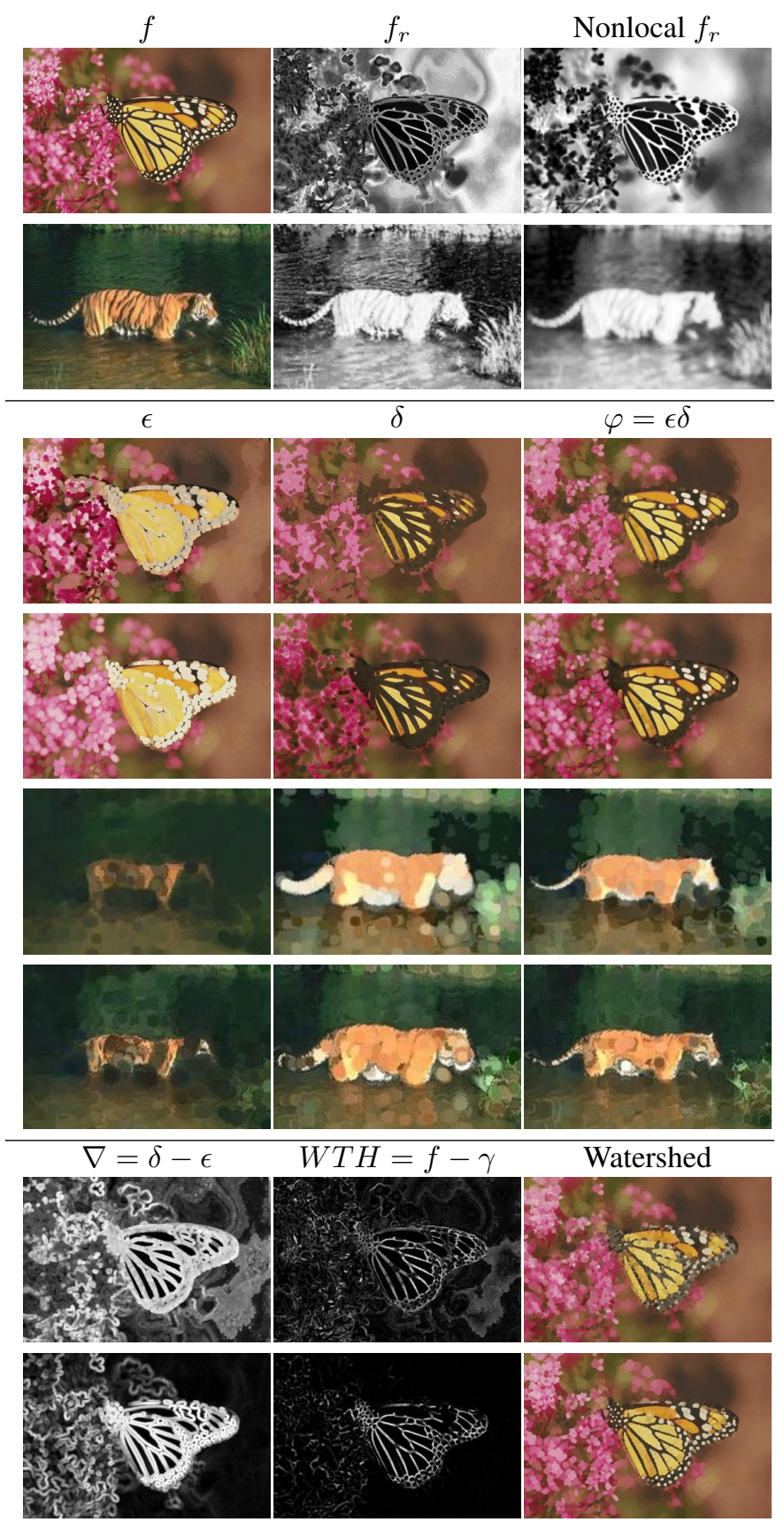

$W T H=f-\gamma$

Watershed
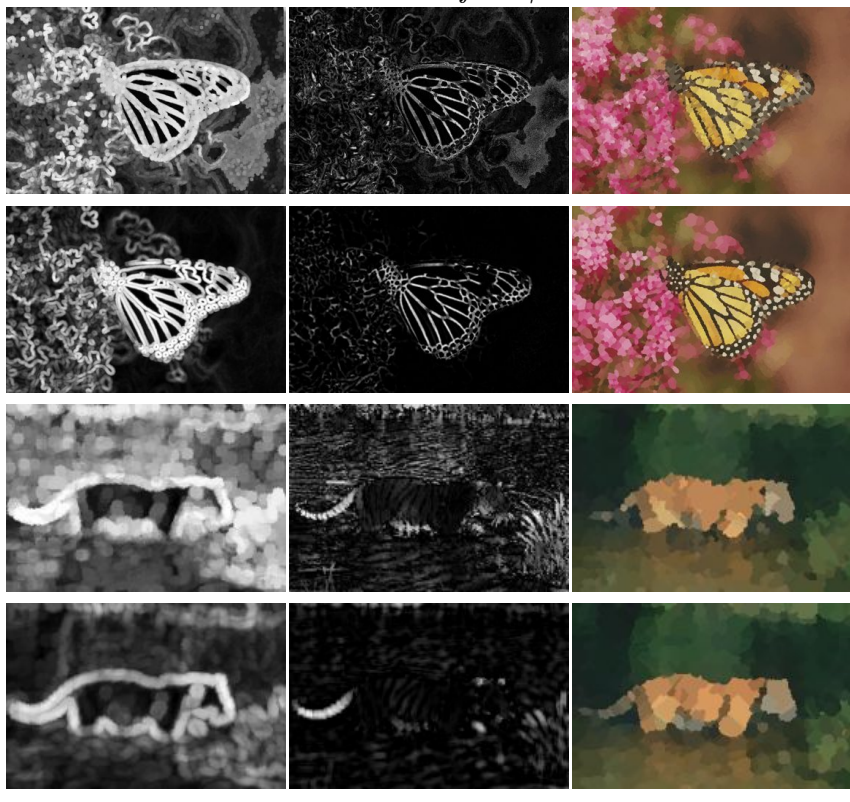

Fig. 2. Morphological processing of a color image in local or nonlocal configurations. The dictionary size is 32 , the structuring element is a circle of radius 5 , patches are $9 \times 9$. See text for details. 